\title{
Diagnostic accuracy of urinary neutrophil gelatinase-associated lipocalin in patients with septic acute kidney injury
}

This article was published in the following Dove Press journal: International Journal of Nephrology and Renovascular Disease II July 2016

Number of times this article has been viewed

\author{
Munna Lal Patel' \\ Rekha Sachan ${ }^{2}$ \\ Radhey Shyam ${ }^{3}$ \\ Satish Kumar' \\ Ritul Kamal ${ }^{4}$ \\ Arvind Misra' \\ 'Department of Medicine, \\ 2Department of Obstetrics \& \\ Gynaecology, ${ }^{3}$ Department of \\ Geriatric Intensive Care Unit, \\ King George Medical University, \\ ${ }^{4}$ Epidemiology Division, Council of \\ Scientific and Industrial Research \\ (CSIR-IITR), Indian Institute of \\ Toxicology Research, Lucknow, Uttar \\ Pradesh, India
}

Correspondence: Munna Lal Patel Department of Medicine, King George Medical University, C-28, Sec-J Aliganj, Lucknow 226024, Uttar Pradesh, India Tel +9l 9839007000

Email patel.ml66@gmail.com
Background: Sepsis is the most common cause of acute kidney injury (AKI). Very few studies have investigated the predictive properties of urinary neutrophil gelatinase-associated lipocalin (uNGAL) as a marker of AKI in septic patients. The aim of this study is to examine uNGAL in septic patients with and without AKI and to evaluate its predictive value.

Methods: We prospectively studied 155 patients with sepsis over a period of 1 year. Urine was analyzed for neutrophil gelatinase-associated lipocalin at 12, 24, and 48 hours after admission. Patients with $<24$-hour stay and those with chronic kidney disease were excluded. AKI was classified according to the Acute Kidney Injury Network guidelines.

Results: The differences in mean change of uNGAL at 12, 24, and 48 hours were $80.00 \pm 7.00$ $\mathrm{ng} / \mathrm{mL}$ and $128.13 \pm 22.46 \mathrm{ng} / \mathrm{mL}$, respectively in septic AKI, and $02.07 \pm 0.80 \mathrm{ng} / \mathrm{mL}$ and $26.13 \pm 15.12 \mathrm{ng} / \mathrm{mL}$, respectively in septic non-AKI. At baseline or 12 hours, the cutoff value of $34.32 \mathrm{ng} / \mathrm{mL}$ had a sensitivity and specificity of 86.36 and 80.60 , respectively and an area under curve of 0.81 (95\% CI: 0.73-0.89) for predicting AKI. At the cutoff value $199.99 \mathrm{ng} / \mathrm{mL}$ sensitivity and specificity of 90.0 and 64.66, respectively and an area under curve of 0.82 (95\% CI, 0.75-0.88) for predicting AKI.

Conclusion: The baseline or 12 -hour uNGAL is highly sensitive but a less specific predictor of AKI in septic patients.

Keywords: septicemia, AKI, urinary NGAL, diagnostic accuracy

\section{Background}

Septic acute kidney injury (AKI) is associated with poor outcome compared to septic patients without AKI, hence sepsis is regarded as the most common trigger of AKI in seriously ill patients. Sepsis leads to AKI in $\sim 50 \%$ of all critically ill patients. ${ }^{1}$ There are various other studies that reported that $40 \%-75 \%$ cases of AKI were associated with sepsis. ${ }^{2-4}$ These patients generally had poor prognosis when compared to septic patients without AKI. ${ }^{5-7}$ The unacceptably high mortality rates are associated with septic AKI and this is explained by an incomplete understanding of the pathophysiology and delay in diagnosis, in spite of using serum creatinine and fractional excretion of sodium. Furthermore, serum creatinine level may take up to 2 days to rise after kidney injury. Therefore, early detection of septic AKI is of great importance to enable adequate treatment in these patients and improve their outcomes. Early diagnosis of AKI remains difficult because it relies on imperfect parameters, such as serum creatinine, while the introduction of new serum and urinary biomarkers could hypothetically allow earlier diagnosis and better prognostication. ${ }^{8,9}$ 
At present, neutrophil gelatinase-associated lipocalin (NGAL) has been the most frequently investigated biomarker for early diagnosis of AKI.$^{10}$ In humans, three different forms of NGAL can be found, namely, a $25 \mathrm{kDa}$ monomer, a $45 \mathrm{kDa}$ dimer, and a $135 \mathrm{kDa}$ heterodimer, covalently conjugated with gelatinase. ${ }^{11-13}$ Yet, no commercially available immunoassays are able to make a clear discrimination between the monomer, mainly released from tubular epithelial cells, and the dimer, originating from neutrophils. ${ }^{12}$ Studies reported that there was a rise in the biomarker level, without a rise in serum creatinine or a decrease in urinary output, which resulted in the speculation of the existence of a new entity called subclinical AKI. ${ }^{14,15}$

The aim of this study was to examine the urinary NGAL (uNGAL) in septic patients with and without AKI. Additional objective was to explore the utility of UNGAL in the prediction of AKI.

\section{Materials and methods}

This was a prospective cohort study carried out in the Department of Medicine, Nephrology Unit at King George Medical University, Lucknow, UP, India, over a period of 1 year from August 2014 to July 2015. The reporting of this study follows the Strengthening the Reporting of Observational Studies in Epidemiology guideline. ${ }^{16}$ After informed written consent and approval from the Institutional Ethic Committee, patients were enrolled in the study. During this period, 18,665 patients were admitted in indoor wards, out of which 170 patients with septicemia fulfilling the inclusion criteria were enrolled in the study. Total subjects were divided into two groups, 88 subjects of septicemia with AKI (group I) and 67 subjects of septicemia without AKI (group II). Fifteen patients dropped out of the study, so are excluded from the analysis (Figure 1).

We included patients in the range of 18-65 years who had sepsis according to the "Survival Sepsis Campaign 2012"17; and patients with end-stage kidney disease (defined as estimated glomerular filtration rate $\leq 15 \mathrm{~mL} / \mathrm{min} / \mathrm{m}^{2}$ ), chronic dialysis therapy, confirmed and/or suspected acute glomerulonephritis, acute interstitial nephritis, renal vasculitis or postrenal chronic kidney disease, and those who had just undergone renal transplantation or were recipients of prior kidney transplant were excluded.

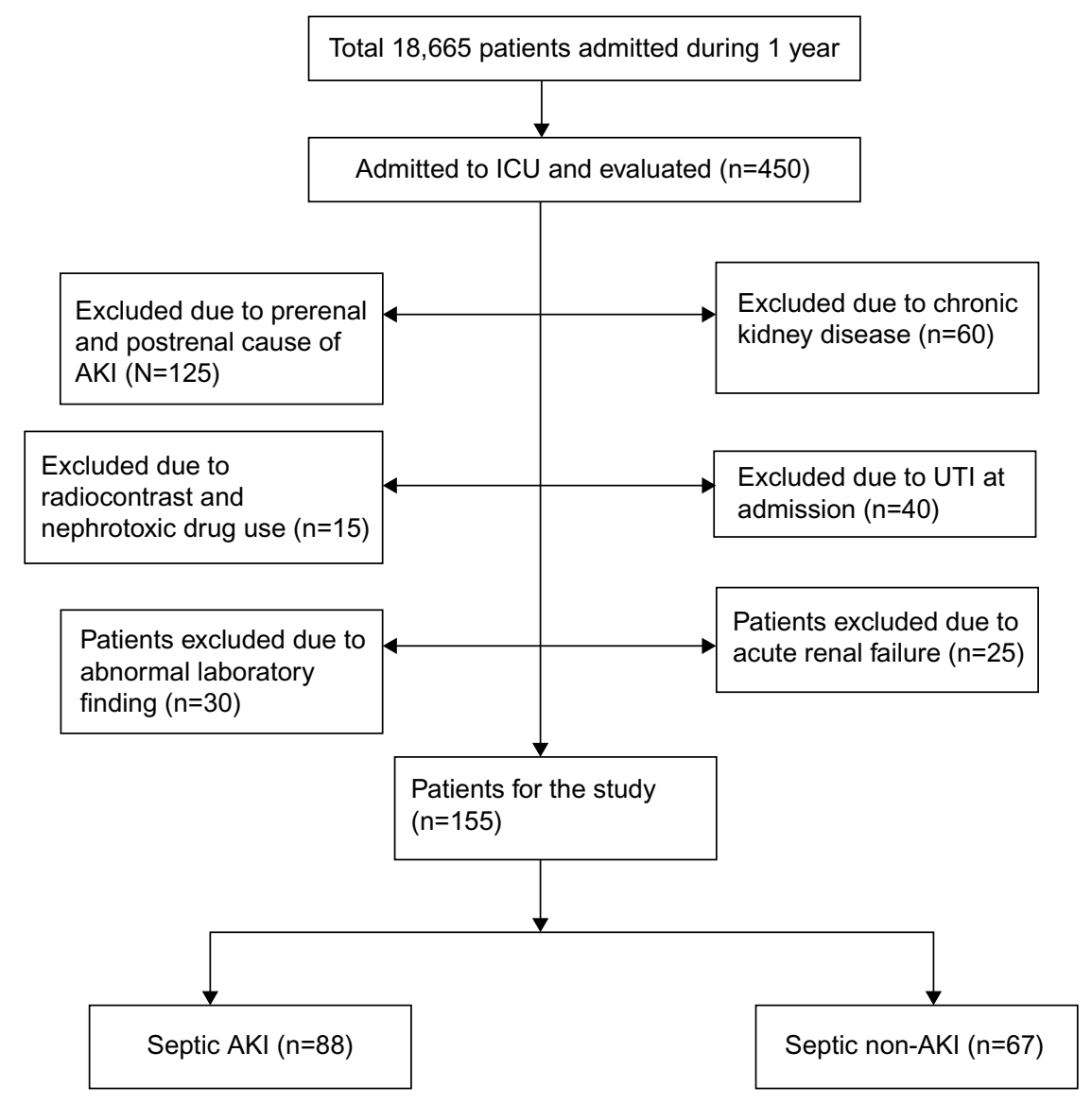

Figure I 18,665 patients admitted during August 20I4-July 2015.

Abbreviations: AKI, acute kidney injury; ICU, intensive care unit; UTI, urinary tract infection. 
AKI was defined and classified according to AKIN criteria. ${ }^{18}$ Worsening AKI was defined as an increase in RIFLE category (from RISK to INJURY, RISK to FAILURE, or INJURY to FAILURE) in the 48 hours after enrollment or renal replacement therapy (RRT) initiation, as previously defined..$^{19}$ Baseline serum creatinine was defined as the lowest creatinine value in the last 6 months before AKI or, for those without this measurement, the lowest value achieved during hospitalization in the absence of dialysis. ${ }^{20}$ Day 0 was defined as the calendar day of admission and thus its length varied depending on the time of presentation. Sepsis syndrome was defined according to the consensus guidelines. ${ }^{21}$ Organ dysfunction/failure was assessed by the Sequential Organ Failure Assessment (SOFA) score, illness severity score (APACHE II). Oliguria was defined as a urine output $<400$ $\mathrm{mL} / 24 \mathrm{~h}$. Vasopressor therapy was defined as the use of any vasopressor or inotropic drugs (dopamine, dobutamine, or noradrenaline) after hospitalization. Shock was defined as a mean arterial pressure $<60 \mathrm{mmHg}$ and/or a need for vasoactive support plus a blood lactate $\geq 4 \mathrm{mmol} / \mathrm{L} .^{22}$

Clinical and laboratory data were collected on standardized data collection forms. Urine was analyzed for NGAL after 12, 24, and 48 hours. Baseline UNGAL was defined as the estimated value of UNGAL, 12 hours from admission. Peak value of UNGAL was defined as the maximum value of uNGAL during the hospital stay, before the development of renal failure. All subjects were admitted to the hospital and monitored daily for biochemical parameters, the length of hospital stay, the need for RRT, and the presence of sepsis. RRT treatment was guided by the physician based upon clinical necessity.

\section{Biochemical analysis}

Five milliliters of venous blood sample was collected once daily for up to 3-5 days. The blood was centrifuged at 5,000 rpm for 10 minutes at room temperature; serum was separated for routine hematology; biochemistry, urinalysis, and urine protein measurements were performed as per study protocols. An automated blood-cell analyzer (BC-5380, Mindray, Guangdong, People's Republic of China) was used for routine hematology testing, and an automated clinical biochemistry analyzer (Cobas C 311 Roche-Hitachi, Japan) was used for blood urea nitrogen, creatinine, uric acid, serum lipids, electrolytes, and albumin.

A clean, morning midstream urine sample $(5 \mathrm{~mL})$ was collected in a sterile test tube and centrifuged at 5,000 rpm for 15 minutes. The supernatant was transferred to an Eppendorf tube and stored at $-80^{\circ} \mathrm{C}$ until assayed for urine NGAL
Urine was analyzed for NGAL at 12, 24, and 48 hours after enrollment. Urine NGAL was measured using the BioVendor Human Lipocalin-2/NGAL ELISA, which is a sandwich enzyme immunoassay for the quantitative measurement of human NGAL as per producer protocol.

\section{Study outcomes}

The primary outcome variable was AKI occurring during the hospital stay, and the secondary outcomes were AKI stage III.

\section{Statistical analysis}

Continuous variables were expressed as mean \pm standard deviation and compared using one-way analysis of variance followed by Tukey's post hoc tests. Correlations between various kidney function parameters have been calculated using Pearson correlation coefficient. Receiver operator curve analysis has been done for NGAL and for identifying the optimal NGAL cutoff values for predicting AKI. Statistical significance was set at $P<0.05$. All the analysis has been done using SPSS 20.0 (IBM Corporation, Armonk, NY, USA) and MedCalc software.

\section{Observation and result}

A total of 155 patients were enrolled in the study out of which $88(56.8 \%)$ were septic AKI patients that included 35 females and 53 males. They were admitted to the hospital $\sim 3-5$ days after the acute onset of clinical symptoms. In septic non-AKI group 67 (43.2\%) were study subjects, out of which 39 were male and 28 were female. The baseline information of patients and laboratory test results was compared between the two groups, septic AKI group vs septic non-AKI group. Study results showed that the length of the hospital stay was higher in the "septic AKI group" when compared to the septic non-AKI group. We found significantly higher APACHE II score and Sequential Organ Failure Assessment score in the septic AKI group as compared to septic non-AKI $(26.84 \pm 6.4$ vs $18.6 \pm 8.4, P<0.001)$ and $(10.6 \pm 2.8$ vs $6.8 \pm 2.4$, $P<0.001$ ), and decreased urine output was also more in the septic AKI group as compared to the septic non-AKI group (844.32 \pm 672.98 vs $1,012.69 \pm 367.46, P=0.06$ ), respectively. The level of UNGAL was high in the septic AKI group as compared to the septic non-AKI group (261.06 \pm 47.91 vs

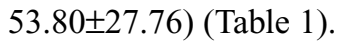

\section{Correlation of UNGAL with other parameters}

The early increasing uNGAL reached its peak after 48 hours of development of AKI followed by consistently increased 
Table I Demographic and biochemical parameters

\begin{tabular}{|c|c|c|c|}
\hline Parameter & Septic non-AKI $(n=67)$ & Septic AKI $(n=88)$ & Significance \\
\hline \multicolumn{4}{|l|}{ Sex } \\
\hline Male, n (\%) & $39(58.21)$ & $53(60.23)$ & $P=0.8$ \\
\hline Female, n (\%) & $28(4 I .79)$ & $35(39.77)$ & \\
\hline \multirow[t]{2}{*}{ Age, years } & $37.88 \pm 16.09$ & $38.98 \pm 14.32$ & $P=0.65$ \\
\hline & $(15-85)$ & $(19-78)$ & \\
\hline \multirow[t]{2}{*}{ Systolic BP mmHg (mean \pm SD) } & $116.16 \pm 22.11$ & $119.66 \pm 27.10$ & $P=0.39$ \\
\hline & $(70-170)$ & $(60-190)$ & \\
\hline \multirow[t]{2}{*}{ Diastolic BP $\mathrm{mmHg}($ mean \pm SD) } & $71.03 \pm 13.97$ & $68.73 \pm 21.58$ & $P=0.45$ \\
\hline & $(50-100)$ & $(40-90)$ & \\
\hline \multirow[t]{2}{*}{ Hospital stay (mean \pm SD) } & $5.33 \pm 1.67$ & $12.5 \pm 4.3$ & $P<0.001$ \\
\hline & $(2-9)$ & $(2-19)$ & \\
\hline \multirow[t]{2}{*}{ Urine output (mean \pm SD) } & $1,012.69 \pm 367.46$ & $844.32 \pm 672.98$ & $P=0.06$ \\
\hline & $(200-2,000)$ & $(100-6,000)$ & \\
\hline \multirow[t]{2}{*}{ Serum creatinine (Baseline) (mean $\pm S D$ ) } & $1.08 \pm 0.24$ & $1.35 \pm 0.30$ & $P<0.001$ \\
\hline & $(0.12-1.54)$ & $(0.12-1.98)$ & \\
\hline \multirow[t]{2}{*}{ Serum creatinine (Peak) (mean \pm SD) } & $1.28 \pm 0.29$ & $4.12 \pm 1.85$ & $P<0.001$ \\
\hline & $(0.19-1.99)$ & $(1.98-11.0)$ & \\
\hline uNGAL (mean \pm SD) & $25.60 \pm 11.84$ & $52.93 \pm 17.45$ & $P<0.001$ \\
\hline (baseline) (ng/mL) & $(5.85-55.0)$ & $(20.66-110.09)$ & \\
\hline $\mathrm{uNGAL}($ mean $\pm \mathrm{SD})$ & $53.80 \pm 27.76$ & $261.06 \pm 47.91$ & $P<0.001$ \\
\hline (peak) (ng/mL) & $(15.22-121.56)$ & $(154-378.89)$ & \\
\hline APACHE II score (mean \pm SD) & $18.6 \pm 8.4$ & $26.84 \pm 6.4$ & $P<0.001$ \\
\hline SOFA score $($ mean $\pm S D)$ & $6.8 \pm 2.4$ & $10.6 \pm 2.8$ & $P<0.001$ \\
\hline
\end{tabular}

Abbreviations: AKI, acute kidney injury; BP, blood pressure; SOFA, Sequential Organ Failure Assessment; uNGAL, urinary neutrophil gelatinase-associated lipocalin.

in the levels of blood urea and serum creatinine in patients developing septic AKI and reaching their maximum value within 7 days. In these patients, uNGAL preceded the peaks of blood urea and serum creatinine by $3-4$ days. The uNGAL correlated positively with the blood urea $(r=0.27, P<0.01)$, serum creatinine $(r=0.44, P<0.001)$, and the length of hospital stay $(r=0.46, P<0.001)$ (Figures 2-4).

\section{AKI progression in groups}

Among septic non-AKI, 57 (85.07\%) patients were at risk, seven $(10.45 \%)$ patients as injury, and only three $(4.48 \%)$ as failure at baseline. After 48 hours, 43 (64.17\%) were in the risk category, 21 (31.34\%) patients in injury, and three (4.4\%) patients were considered as failure. Only 14 (20.9\%) patients progressed from risk to injury. There was no significant change in the AKIN criteria within the septic non-AKI group after 48 hours, and no significant difference was observed between the two groups $(P<0.001)$. But in the septic AKI group, five $(5.68 \%)$ patients were at "risk", 46 (51.13\%) patients in "injury", and 37 (42.04\%) patients in "failure" category as per the AKIN criteria at baseline. After 48 hours, eight (9\%) were in the risk category, $36(40.9 \%)$ patients in injury category, and $44(50 \%)$ patients were considered as failure. There was a significant change in AKI stages within septic AKI after 48 hours $(P<0.001)$ (Table 2).

\section{Mean changes in uNGAL from baseline}

In the septic non-AKI group, difference in mean change of uNGAL between 12-hour and 24-hour values was $02.07 \pm 0.80$ $\mathrm{ng} / \mathrm{mL}$ and difference between 24-hour and 48-hour values was $26.13 \pm 15.12 \mathrm{ng} / \mathrm{mL}$. In the septic AKI group, difference in mean change of UNGAL between 12-hour and 24-hour values was $80.00 \pm 7.00 \mathrm{ng} / \mathrm{mL}$, and difference between 24-hour and 48 -hour values was $128.13 \pm 22.46 \mathrm{ng} / \mathrm{mL}$. Within group analysis, difference was statistically highly significant $(P<0.0001)$ in septic AKI but not in septic non-AKI group $(P<0.01)$. On evaluating between group analysis, we found highly significant difference in septic AKI at 12 and 48 hours as compared to septic non-AKI $(P<0.0001)$ (Table 3$)$.

\section{Sensitivity and specificity of UNGAL with septic AKI}

The 12-hour value and peak (48 hours) uNGAL were significantly higher in patients with septic AKI than those with septic non-AKI (Table 1). The accuracy for the prediction of septic $\mathrm{AKI}$, as quantified by the area under the receiver-operating characteristic curve (AU-ROC), was significantly higher with the peak UNGAL than that with the 12-hour uNGAL. AU-ROC for the peak uNGAL: 0.82 (95\% CI, 0.75-0.88) vs AU-ROC for the baseline uNGAL: 0.81( $95 \%$ CI: 0.73-0.89) 


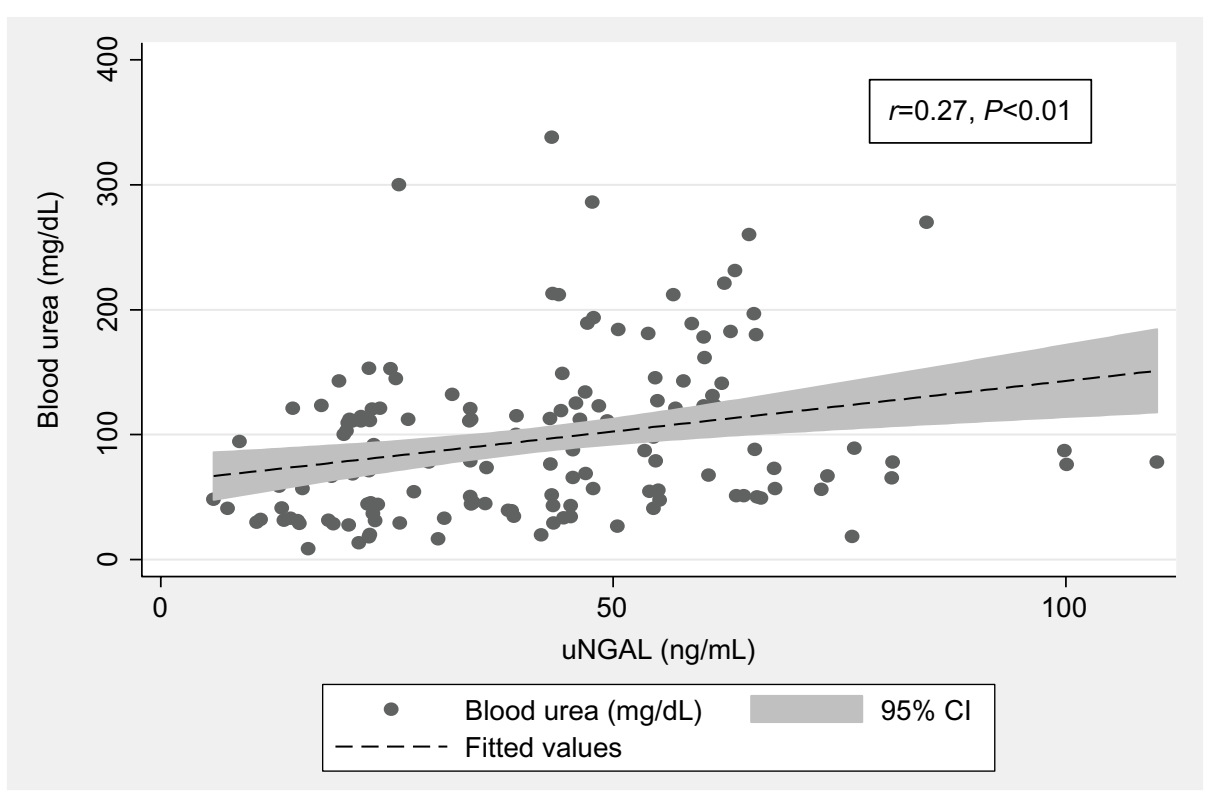

Figure 2 Correlation of baseline UNGAL with levels of blood urea.

Notes: Significant positive correlation was observed between blood urea and uNGAL values with correlation coefficient, $r=0.27$ ( $P<0.01)$. An increase in the value of uNGAL was associated with the increase in the levels of blood urea.

Abbreviations: $\mathrm{Cl}$, confidence interval; uNGAL, urinary neutrophil gelatinase-associated lipocalin.

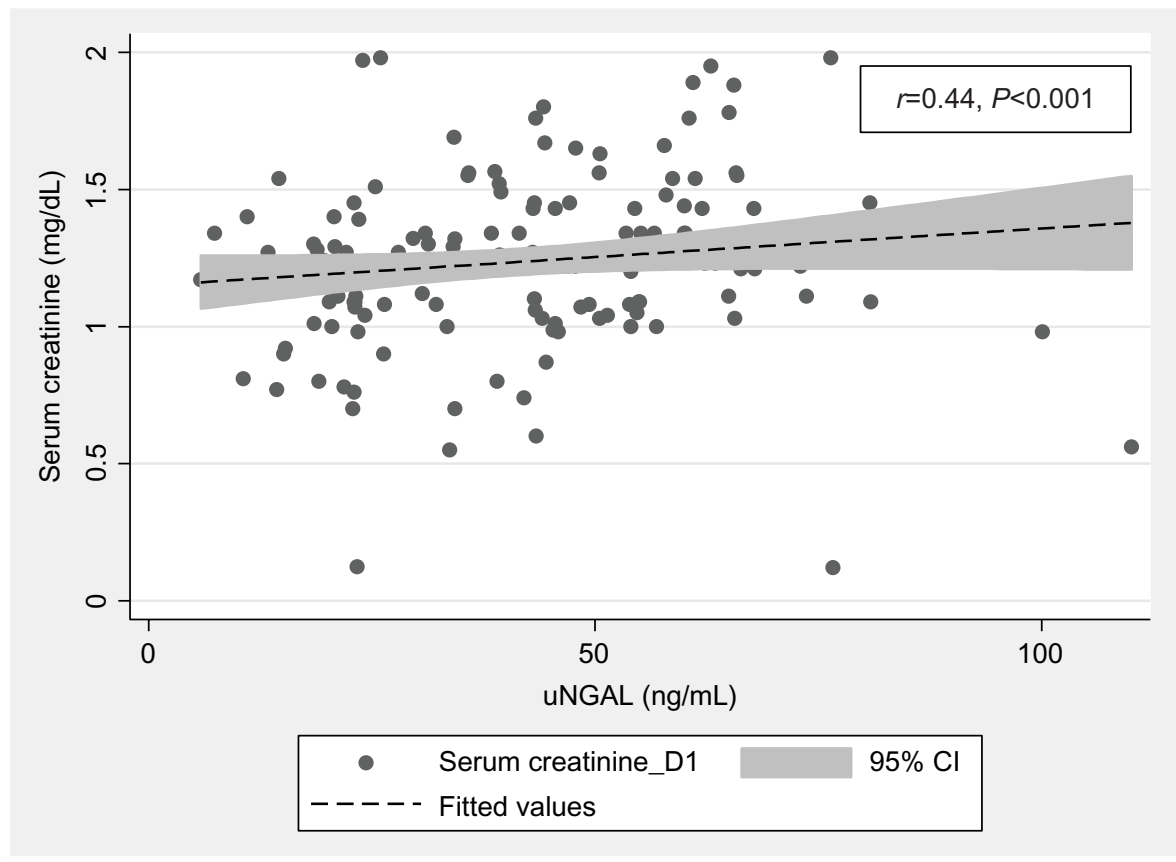

Figure 3 Correlation of baseline uNGAL with levels of serum creatinine.

Notes: A positive significant correlation was observed between uNGAL and serum creatinine at baseline. The correlation coefficient was 0.44 ( $P<0.00 \mathrm{I}$ ).

Abbreviations: $\mathrm{Cl}$, confidence interval; uNGAL, urinary neutrophil gelatinase-associated lipocalin.

(Figure 5). At 12 hours, the cutoff value of $34.32 \mathrm{ng} / \mathrm{mL}$ had a sensitivity and specificity of 86.36 and 80.60 , respectively and an area under curve of 0.81 (95\% CI: $0.73-0.89)$ for predicting the AKI. At peak or at 48 hours, the cutoff value of $199.99 \mathrm{ng} / \mathrm{mL}$ had a sensitivity and specificity of 90.0 and 64.66, respectively and an area under curve of 0.82 (95\% CI, 0.75-0.88) for predicting AKI (Table 4; Figure 5).

\section{Discussion}

In our study, clinical courses of patients with sepsis were prospectively followed throughout hospitalization. AKI is an important cause of morbidity and mortality in seriously ill patients. Our study results showed that the length of hospital stay was significantly higher in "septic AKI group" when compared to "septic non-AKI group". This result emphasizes 


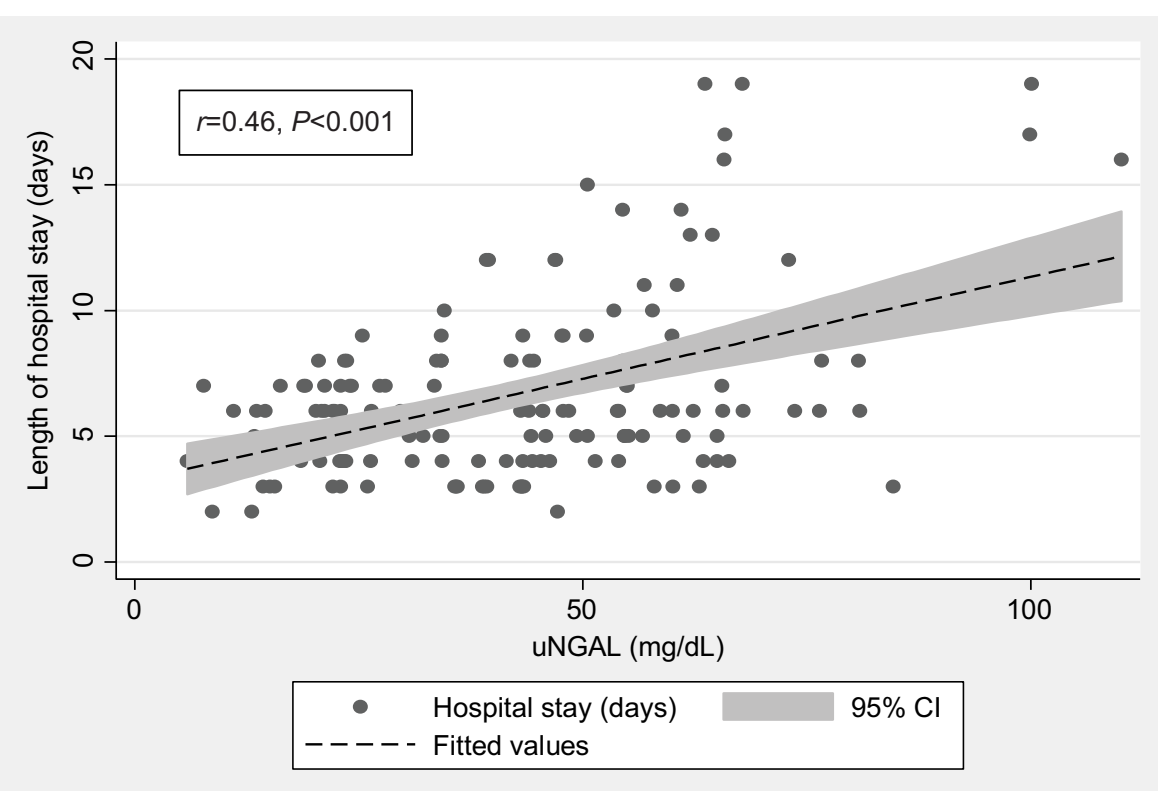

Figure 4 Correlation of baseline uNGAL with the length of hospital stay (in days).

Notes: Significantly positive correlation was observed between uNGAL and the duration of hospital stay with correlation coefficient, $r=0.46$ ( $P<0.00 \mathrm{I})$. An increase in the levels of UNGAL was associated with increased duration of hospital stay of the subjects.

Abbreviations: $\mathrm{Cl}$, confidence interval; uNGAL, urinary neutrophil gelatinase-associated lipocalin.

Table $2 \mathrm{AKI}$ progression in groups

\begin{tabular}{|c|c|c|c|c|c|c|}
\hline \multirow[t]{2}{*}{ Stage } & \multicolumn{3}{|c|}{ Septic non-AKI $(n=67)$} & \multicolumn{3}{|c|}{ Septic AKI $(n=88)$} \\
\hline & Baseline & After 48 hours & $P$-value & Baseline & After 48 hours & $P$-value \\
\hline & $N(\%)$ & $N(\%)$ & & $N(\%)$ & $N(\%)$ & \\
\hline Risk (I) & $57(85.07)$ & $43(64.17)$ & $P<0.001$ & $5(5.68)$ & $8(9.0)$ & $P<0.001$ \\
\hline Injury (II) & $7(10.45)$ & $21(31.34)$ & $P<0.00$ I & $46(51.13)$ & $36(40.9)$ & $P<0.001$ \\
\hline Failure (III) & $3(4.48)$ & $3(4.4)$ & $P<0.001$ & $37(42.04)$ & $44(50.0)$ & $P<0.00$ I \\
\hline
\end{tabular}

Notes: Risk: serum creatinine increases to 1.5 -fold from baseline. Injury: serum creatinine increases to 2.0 -fold from baseline. Failure: serum creatinine increases to 3.0 -fold from baseline.

Abbreviation: AKI, acute kidney injury.

Table 3 Mean changes in uNGAL from baseline

\begin{tabular}{|c|c|c|c|c|c|c|}
\hline uNGAL (ng/mL) & At 12 hours & At 24 hours & $\begin{array}{l}\text { Difference } \\
\text { between I 2- and } 24 \\
\text { hour values }\end{array}$ & At 48 hours & $\begin{array}{l}\text { Difference } \\
\text { between } 24 \text {-hour } \\
\text { and } 48 \text {-hour values }\end{array}$ & $\begin{array}{l}P \text {-value within } \\
\text { groups }\end{array}$ \\
\hline Septic non-AKI, ng/mL & $25.60 \pm 11.84$ & $27.67 \pm 12.64$ & $02.07 \pm 0.80$ & $53.80 \pm 27.76$ & $26.13 \pm 15.12$ & $P<0.01$ \\
\hline Septic AKI, ng/mL & $52.93 \pm 17.45$ & $132.93 \pm 24.45$ & $80.00 \pm 7.00$ & $261.06 \pm 47.91$ & $128.13 \pm 22.46$ & $P<0.000 I$ \\
\hline$P$-value between groups & $P<0.000$ I & $P<0.000$ I & & $P<0.0001$ & & \\
\hline
\end{tabular}

Note: Data is presented as mean \pm SD.

Abbreviations: AKI, acute kidney injury; uNGAL, urinary neutrophil gelatinase-associated lipocalin.

the importance of early detection of septic AKI, especially in emergency departments and intensive care units (ICUs). During the course of sepsis, the patients gradually progress from mild-to-severe stages of AKI. In these patients, the peak uNGAL positively correlated with the levels of blood urea, serum creatinine, and the length of hospital stay, reflecting the severity of renal dysfunction.

Approximately $90 \%$ of the patients in the study of sepsis had increased UNGAL, but this value was markedly increased in septic AKI group. These patients also had a higher level of blood urea, serum creatinine, and increased progression of AKI. uNGAL was also measured in one study of sepsis in which $63(42 \%)$ patients out of 151 patients had increased uNGAL. ${ }^{23}$

Haase-Fielitz et al ${ }^{24}$ studied adult cardiac surgery patients and they reported that predictive value of plasma NGAL increases with increasing grade of AKI.

Similarly, a recent study in a cohort of 45 patients of septicemia, showed that uNGAL was a good diagnostic marker for AKI, but this did not indicate about the severity of AKI 


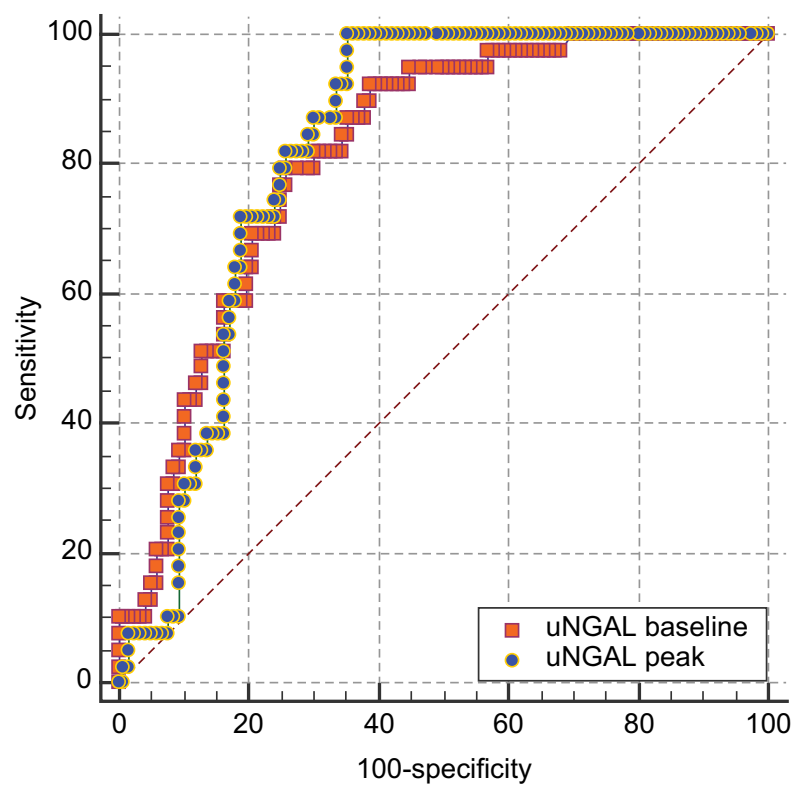

Figure 5 ROC analysis for predicting the diagnostic accuracy of urinary NGAL for AKI.

Notes: At baseline, the cutoff value $34.32 \mathrm{ng} / \mathrm{mL}$ had a sensitivity and specificity of 86.36 and 80.60 , respectively and an area under curve of $0.81(95 \% \mathrm{Cl}, 0.73-0.89)$ for predicting AKI. At peak, the cutoff value $199.99 \mathrm{ng} / \mathrm{mL}$ had a sensitivity and specificity of 90.0 and 64.66 , respectively and an area under curve of $0.82(95 \% \mathrm{Cl}$, $0.75-0.88$ ) for predicting AKI.

Abbreviations: AKI, acute kidney injury; ROC, receiver operator curve; UNGAL, urinary neutrophil gelatinase-associated lipocalin.

Table 4 Diagnostic accuracy of uNGAL for AKI

\begin{tabular}{lll}
\hline Parameters & uNGAL (baselinel & uNGAL (peakl \\
& I 2 hours), ng/mL & 48 hours), ng/mL \\
\hline Sensitivity & 86.36 & 90.0 \\
Specificity & 80.60 & 64.66 \\
AUC & $0.81(0.73-0.89)$ & $0.82(0.75-0.88)$ \\
Best cutoff value & 34.32 & 199.99 \\
\hline
\end{tabular}

Abbreviations: AKI, acute kidney injury; AUC, area under curve; uNGAL, urinary neutrophil gelatinase-associated lipocalin.

or disease progression, when it was evaluated, after a rise in serum creatinine. ${ }^{25,26}$

In our study, ten patients (11.4\%) did not progress beyond the AKIN stage II, indicating that some patients in this group probably had functional renal failure (transient AKI or prerenal azotemia) without any significant renal tubular injury. The incidence of transient AKI was similar to the study done by Hoste et $\mathrm{al}^{27}(18 \%)$. In such situations, serum creatinine may be elevated due to decreased glomerular filtration rate, but without elevation in UNGAL due to the absence of significant tubular injury. ${ }^{28}$

The diagnostic accuracy of septic AKI was highest with the peak uNGAL at 48 hours, as compared to the baseline or 12-hour uNGAL (Figure 5). Although there is no such huge difference between the two values, the specificity of baseline or 12-hour UNGAL was high at a lower cutoff value. In order to diagnose AKI before rise in serum creatinine, baseline or 12-hour uNGAL can be used in the ICU. Our study result showed that baseline uNGAL was highly specific for the diagnosis of AKI with an AU-ROC of 0.81 .

A study done by Constantin et $\mathrm{al}^{29}$ in the adult population observed that plasma NGAL at ICU admission is an early biomarker of AKI with sensitivity of $85 \%$ and specificity of $97 \%$ with an AUC equivalent of 0.956 (0.864-0.992). Our results are quite similar to the study done by Nickolas et $\mathrm{al}^{28}$ who reported excellent prediction of AKI by uNGAL (AUC of ROC $=0.95)$ with high sensitivity (0.90) and specificity (0.99) of a single measure of UNGAL done during emergency. Increased concentration of NGAL has been found in patients with acute infection as well as in bronchoalveolar lavage fluids with lung disease. Therefore, it is not surprising that the authors found increasing levels of UNGAL in septic patients without AKI. ${ }^{30}$ Our study result was also supported by Wheeler et $\mathrm{al}^{31}$ who reported increasing levels of serum NGAL in critically ill children with systemic inflammatory response syndrome (SIRS) vs septic shock, even in the absence of AKI. So in our study, we preferred to measure NGAL in urine and in septic non-AKI patients also. Martensson et $\mathrm{a}^{13}$ also supported our study that plasma NGAL was raised in patients with SIRS, severe sepsis, and septic shock and should be used with caution as a marker of AKI in ICU patients with septic shock. We also suggested that UNGAL is more useful in predicting AKI, as the levels are not elevated in septic patients without AKI. ${ }^{32}$ In our study at 12 hours uNGAL was significantly higher in those who developed AKI after admission than those who never developed AKI, indicating that UNGAL is indeed an early marker of AKI. Hence, despite limitation in a clinical setting uNGAL can still be a useful early prognostic marker of AKI.

Though the peak level of uNGAL was reached at 48 hours, even at 12 hours after the development of septicemia uNGAL had good diagnostic value; at 12 hours its sensitivity was $86.36 \%$ and specificity was $80.60 \%$ for prediction of AKI. Though the peak value at 48 hours, at the cutoff $199.99 \mathrm{ng} / \mathrm{mL}$ had high sensitivity of $90 \%$, specificity was less than at 12-hour value. Thus, we can say it is good to measure uNGAL even at 12 hours for prediction of AKI.

Variability in the prediction of AKI by uNGAL could be attributed to the type, timing, and nature of renal injury. It is very common to see critically ill patients being admitted to tertiary referral centers late in the course of their illness in India, when AKI has already been established at the time of admission. The high cost of ICU carries a major deterrent 
for self-paying patients, resulting in delayed admission to ICU. Thus, we can say that early detection of septic AKI helps in early interventions, early antibiotic initiation, fluid resuscitation, and restriction of nephrotoxic antibiotics and intravenous contrast dye use.

The level of UNGAL may make it possible to explain the prognosis of septic AKI and the need for RRT on the basis of daily measurements. There are a few limitations to our study. First, this study was relatively small and prospective in nature, our findings may be prone to type II errors, and therefore, these markers require validation in independent cohorts. Second, the early abnormalities, such as hypoalbuminemia, elevated hematocrit, and elevated aspartate and alanine aminotransferase indicating the presence of liver impairment were also noted in the sepsis, but we have not included these in the analysis.

\section{Conclusion}

The increased urinary NGAL is a significant early and consistent laboratory abnormality in septic AKI. It could be having a potential value in risk stratification of patients for the timely initiation of RRT. Its utility is markedly limited where referral is late or when AKI has already been established at the time of admission.

\section{Disclosure}

The authors report no conflicts of interest in this work.

\section{References}

1. Uchino S, Kellum JA, Bellomo R, et al. Acute renal failure in critically ill patients: A multinational, multicenter study. JAMA. 2005;294(7): 813-818.

2. Neveu H, Kleinknecht D, Brivet F, Loirat P, Landais P. Prognostic factors in acute renal failure due to sepsis. Results of a prospective multicenter study. The French study Group on Acute Renal Failure. Nephrol Dial Transplant. 1996;11(2):293-299.

3. Silvester W, Bellomo R, Cole L. Epidemiology, management, and outcome of severe acute renal failure of critical illness in Australia. Crit Care Med. 2001;29(10):1910-1915.

4. Bagshaw SM, Laupland KB, Doig CJ, et al. Prognosis for long-term survival and renal recovery in critically ill patients with severe acute renal failure: A population-based study. Crit Care. 2005;9(6):R700-R709.

5. Bagshaw SM, Uchino S, Bellomo R, et al. Septic acute kidney injury in critically ill patients: Clinical characteristics and outcomes. Clin J Am Soc Nephrol. 2007;2(3):431-439.

6. Oppert M, Engel C, Brunkhorst FM, et al. Acute renal failure in patients with severe sepsis and septic shock - A significant independent risk factor for mortality: Results from the German Prevalence Study. Nephrol Dial Transplant. 2008;23(3):904-909.

7. Bagshaw SM, George C, Bellomo R; ANZICS Database Management Committee. Early acute kidney injury and sepsis: A multicentre evaluation. Crit Care. 2008;12(2):R47.

8. Doi K, Yuen PS, Eisner C, et al. Reduced production of creatinine limits its use as marker of kidney injury in sepsis. J Am Soc Nephrol. 2009;20:1217-1221.
9. Endre ZH, Westhuyzen J. Early detection of acute kidney injury: emerging new biomarkers. Nephrology (Carlton). 2008;13:91-98.

10. Haase M, Bellomo R, Devarajan P, Schlattmann P, Haase-Fielitz A; NGAL Meta-analysis Investigator Group. Accuracy of neutrophil gelatinase-associated lipocalin (NGAL) in diagnosis and prognosis in acute kidney injury: a systematic review and meta-analysis. $\mathrm{Am} \mathrm{J}$ Kidney Dis. 2009;54:1012-1024.

11. Cai L, Rubin J, Han W, Venge P, Xu S. The origin of multiple molecular forms in urine of HNL/NGAL. Clin J Am Soc Nephrol. 2010;5: 2229-2235.

12. Glassford NJ, Schneider AG, Xu S, et al. The nature and discriminatory value of urinary neutrophil gelatinase-associated lipocalin in critically ill patients at risk of acute kidney injury. Intensive Care Med. 2013;39:1714-1724.

13. Martensson J, Xu S, Bell M, Martling CR, Venge P. Immunoassays distinguishing between HNL/NGAL released in urine from kidney epithelial cells and neutrophils. Clin Chim Acta. 2012;413:1661-1667.

14. Haase M, Kellum JA, Ronco C. Subclinical AKI - an emerging syndrome with important consequences. Nat Rev Nephrol. 2012;8:735-739.

15. Ronco C, Kellum JA, Haase M. Subclinical AKI is still AKI. Crit Care. 2012;16:313.

16. von Elm E, Altman DG, Egger M, Pocock SJ, Gøtzsche PC, Vandenbroucke JP; STROBE Initiative. The Strengthening the Reporting of Observational Studies in Epidemiology (STROBE) statement: Guidelines for reporting observational studies. Ann Intern Med. 2007;147: 573-577.

17. Dellinger RP, Levy MM, Rhodes A, et al. Surviving sepsis campaign: international guidelines for management of severe sepsis and septic shock, 2012. Intensive Care Med. 2013;39(2):165-228.

18. Mehta RL, Kellum JA, Shah SV, et al. Acute Kidney Injury Network: report of an initiative to improve outcomes in acute kidney injury. Crit Care. 2007;11:R31.

19. Gammelager H, Christiansen CF, Johansen MB, Tønnesen E, Jespersen B, Sørensen HT. Five-year risk of end-stage renal disease among intensive care patients surviving dialysis-requiring acute kidney injury: a nationwide cohort study. Crit Care. 2013;17(4):R145.

20. Siew ED, Matheny ME, Ikizler TA, et al. Commonly used surrogates for baseline renal function affect the classification and prognosis of acute kidney injury. Kidney Int. 2010;77(6):536-542.

21. Levy MM, Fink MP, Marshall JC, et al. 2001 SCCM/ESICM/ ACCP/ ATS/SIS International Sepsis Definitions Conference. Crit Care Med. 2003;29(4):530-538.

22. Bagshaw SM, Haase M, Haase-Fielitz A, Bennett M, Devarajan P, Bellomo R. A prospective evaluation of urine microscopy in septic and non-septic acute kidney injury. Nephrol Dial Transplant. 2012;27(2):582-588.

23. Aydogdu M, Gürsel G, Sancak B, et al. The use of plasma and urine neutrophil gelatinase associated lipocalin (NGAL) and cystatin $\mathrm{C}$ in early diagnosis of septic acute kidney injury in critically ill patients. Dis Markers. 2013;34(4):237-246.

24. Haase-Fielitz A, Bellomo R, Devarajan P, et al. The predictive performance of plasma neutrophil gelatinase-associated lipocalin (NGAL) increases with grade of acute kidney injury. Nephrol Dial Transplant. 2009;24(11):3349-3354.

25. De Geus HR, Woo JG, Wang Y, et al. Urinary neutrophil gelatinaseassociated lipocalin measured on admission to the intensive care unit accurately discriminates between sustained and transient acute kidney injury in adult critically ill patients. Nephron Extra. 2011;1(1):9-23.

26. Smertka M, Wroblewska J, Suchojad A, et al. Serum and urinary NGAL in septic newborns. Biomed Res Int. 2014;2014:article ID 717318.

27. Hoste EA, Clermont G, Kersten A, et al. RIFLE criteria for acute kidney injury are associated with hospital mortality in critically ill patients: A cohort analysis. Crit Care. 2006;10:R73.

28. Nickolas TL, O'Rourke MJ, Yang J, et al. Sensitivity and specificity of a single emergency department measurement of urinary neutrophil gelatinase-associated lipocalin for diagnosing acute kidney injury. Ann Intern Med. 2008;148:810-819. 
29. Constantin JM, Futier E, Perbet S, et al. Plasma neutrophil gelatinaseassociated lipocalin is an early marker of acute kidney injury in adult critically ill patients: a prospective study. J Crit Care. 2010;25(1): 176.e1-6.

30. Xu S, Venge P. Lipocalins as biochemical markers of disease. Biochim Biophys Acta. 2000;1482(1-2):298-307.
31. Wheeler DS, Devarajan P, Ma Q, et al. Serum neutrophil gelatinase-associated lipocalin (NGAL) as a marker of acute kidney injury in critically ill children with septic shock. Crit Care Med. 2008;36(4):1297-1303.

32. Mårtensson J, Bell M, Oldner A, Xu S, Venge P, Martling CR. Neutrophil gelatinase-associated lipocalin in adult septic patients with and without acute kidney injury. Intensive Care Med. 2010;36(8):1333-1340.
The International Journal of Nephrology and Renovascular Disease is an international, peer-reviewed open access journal focusing on the pathophysiology of the kidney and vascular supply. Epidemiology, screening, diagnosis, and treatment interventions are covered as well as basic science, biochemical and immunological studies. The manuscript management system is completely online and includes a very quick and fair peer-review system, which is all easy to use. Visit http://www dovepress.com/testimonials.php to read real quotes from published authors.

Submit your manuscript here: https://www.dovepress.com/international-journal-of-nephrology-and-renovascular-disease-journal 